\title{
QUANTUM STATES AND NUMBER-PHASE UNCERTAINTY RELATIONS MEASURED BY OPTICAL HOMODYNE TOMOGRAPHY
}

\author{
M.G. Raymer, D.T. Smithey, M. Beck AND J. Cooper*
}

Department of Physics and Chemical Physics Instilute, University of Oregon Eugene, OR, 97403, USA

Experiments have been performed to determine the Wigner distribution and the density matrix (and for pure states the wave function) of a light mode, by using tomographic inversion of a set of measured probability distributions for quadrature amplitudes. From these measurements the quantum distributions of optical phase and photon number have been obtained. The measurements of quadrature-amplitude distributions for a temporal mode of the electromagnetic field are carried out using balanced homodyne detection. We refer to this new method as optical homodyne tomography. Given the measured density matrix, one can experimentally infer any of the various quantum distributions of optical phase, in particular the Pegg-Barnett (or, equivalently, Shapiro-Shepard) phase distribution, the marginal Wigner distribution, and the Vogel-Schleich operational phase distribution. We have used this approach to make measurements of the number-phase uncertainty relation for coherent-state fields. The coherent states do not attain the minimum value for the number-phase uncertainty product, as set by the expectation value of the commutator of the number and phase operators; this is true theoretically and experimentally.

PACS numbers: $42.50 . \mathrm{Wm}, 03.65 . \mathrm{Bz}$

\section{Introduction}

In quantum optics, measurements have usually focussed on determining values of one or more particular physical quantities, or observables, for example photon number, field phase, or quadrature field amplitude. Another approach towards characterizing a physical system (actually an ensemble of similar systems) is to determine fully its quantum state. Due to a lack of practical means for doing so, this idea has not seen a lot of attention until recently. Here we review several state-characterizing measurements we have carried out using the technique of "optical homodyne tomography" [1, 2]. Further, we discuss the kinds of information,

* Permanent address: Joint Institute for Laboratory Astrophysics and the Department of Physics, University of Colorado and the National Institute of Standards and Technology, Boulder CO 80309, USA. 
such as phase and photon number distributions, which are available from such a state measurement [3]. From such distributions the number-phase uncertainty relations can be studied experimentally [4].

Recently it was pointed out by Vogel and Risken that, for a single-mode light field, knowledge of an infinite and continuous (uncountable) sct of quadrature-amplitude distributions is equivalent to full knowledge of the density matrix operator [5]. They pointed out that the Wigner distribution, which has a one-to-one relation to the density operator, is related to this set of distributions by a projection integral in a rotated frame (the Radon transform). Further, they showed that in principle these distributions can be measured for a mode of the clectromagnetic field by using coherent optical detection (sce below). Our measurements of the state of a light mode are based on this idea, and take advantage of the following:

1) The Radon transform is the same as that underlying the principles of tomographic reconstruction, familiar in medical imaging. There it is known that a finite set of one-dimensional distributions (projections) is sufficient to reconstruct a two-dimensional density function by using the numerical algorithms known as computer-aided tomography (CAT) [6]. The density function thus reconstructed is unique, but is a smoothed, or filtered, version of the true underlying function. The finer the detail that is desired, the larger the number of distributions that must be measured.

2) Recent advances in optical detection techniques [7] have made possible the measurement of the needed distributions.

In essence, the method is this: make repeated measurements of quadrature amplitude on an ensemble of similarly prepared light pulses; from these determine the distributions of these quadratures for many different valucs of local oscillator phase (this corresponds to different llilbert-space representations); carry out the inverse Radon transform on the set of distributions to determine the Wigner function; from this delermine the wave function and/or the density matrix.

This paper reviews the basis of tomographic state determination, and its applications to measuring phase distributions, and also gives two new results: the measurement of the Vogel-Schleich operational phase distribution, and the comparison of two different methods (Eqs. (12) and (14)) for calculating the number-phase uncertainty lower bound from experimental data.

\section{Optical homodyne tomography $[1,2]$}

A balanced homodyne detector makes measurements that correspond to the quadrature operator $\widehat{x}_{\theta}$ of the signal field, and that quadrature which is measured is determined by the phase $\theta$ of a "local-oscillator" flid. The set up is shown schematically in Fig. 1 . The signal field $\widehat{E}(t)$ (whose state is to be determined) is superposed on a $50 / 50$ beam splitter with the local-oscillator field $\widehat{E}_{\mathrm{LO}}(t)$, having the same carrier frequency $\omega$. The electric-field operator of the signal mode is

$$
\begin{aligned}
\widehat{E}(t) & =E_{0}(t)[\widehat{x} \cos (\omega t)+\widehat{p} \sin (\omega t)] \\
& =E_{0}(t)\left[\widehat{x}_{\theta} \cos (\omega t+\theta)+\widehat{p}_{\theta} \sin (\omega t+\theta)\right],
\end{aligned}
$$

where $\omega$ is the optical frequency and $E_{0}(t)$ corresponds roughly to the electric-field strength of a pulse containing a single photon (an operator is denoted by a caret). 


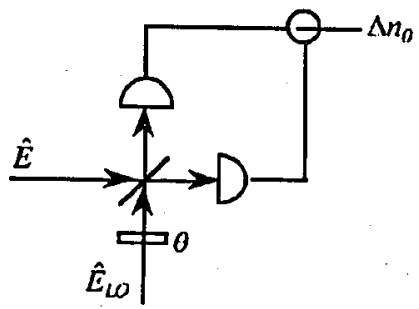

Fig. 1. Set up for optical homodyne detection.

The quadrature operators $\widehat{x}, \widehat{p}$ are related to creation and annihilation operators for a particular spatial-temporal mode by $\widehat{x}=\left(\hat{a}+\hat{a}^{\dagger}\right) / \sqrt{2}$ and $\hat{p}=\left(\widehat{a}-\hat{a}^{\dagger}\right) / i \sqrt{2}$, and obey $[\widehat{x}, \hat{p}]=\mathrm{i}$. The rotated quadrature operators are defined as

$$
\begin{aligned}
& \widehat{x}_{\theta}=\widehat{x} \cos \theta+\widehat{x} \sin \theta, \\
& \widehat{p}_{\theta}=-\widehat{x} \sin \theta+\widehat{p} \cos \theta,
\end{aligned}
$$

for an arbitrary angle $\theta$. This is illustrated in Fi.r. 2. The local-oscillator field is

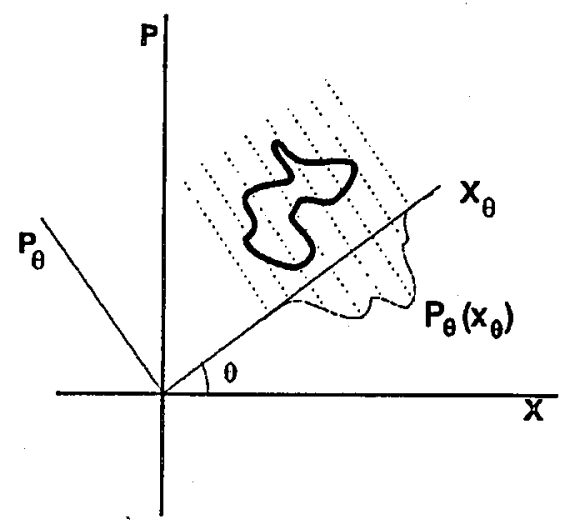

Fig. 2. The rotated coordinate system. This also shows the relationship between the Wigner function (bold line) and the quadrature distributions $P_{\theta}\left(x_{\theta}\right)$.

much stronger than the signal field, and is in a large-amplitude coherent state, and so its operator can be approximated by a classical field, with phase $\theta$ determined by the device labelled $\theta$,

$$
\widehat{E}_{\mathrm{LO}}(t)=E_{\mathrm{LO}}(t) \cos (\omega t+\theta) \text {, }
$$

where $E_{\mathrm{LO}}(t)$ is a $c$-number function. The spatial-temporal mode of the signal field which is selected by the homodyne detector is the same as that of the local oscillator. The superposition fields are both detected by high-efficiency $(80 \%)$ photodiodes and the resulting photocurrents are subtracted and integrated. The difference photoelectron number $\Delta \hat{n}_{\theta}$ thus measured is proportional to the desired quadrature amplitude

$$
\Delta \hat{n}_{\theta} \propto \int\left[\left(\widehat{E}_{\mathrm{LO}}(t)+\widehat{E}(t)\right)^{2}-\left(\widehat{E}_{\mathrm{LO}}(t)-\widehat{E}(t)\right)^{2}\right] \mathrm{d} t \propto \widehat{x}_{\theta}
$$


To normalize this expression correctly the mean photoelectron number $\bar{n}_{\text {LO }}$ produced by the local oscillator pulse must be measured. The relation is $[1,2]$ :

$$
\widehat{x}_{\theta}=\Delta \widehat{n}_{\theta} / \sqrt{2 \vec{n}_{\mathrm{LO}}} \text {. }
$$

Thus for a given $\theta$, measurements of photoelectron number yield measurements of quadrature amplitude. By making thousands of such measurements under identical conditions the probability density $P_{\theta}\left(x_{\theta}\right)$ in the form of histograms can be determined.

For a single light mode described by $\widehat{x}$ and $\widehat{p}$, the Wigner distribution corresponding to a particular state with density operator $\hat{\rho}$ is [8]:

$$
W(x, p)=\frac{1}{\pi} \int_{-\infty}^{\infty}\left\langle x+x^{\prime}|\hat{\rho}| x-x^{\prime}\right\rangle \exp \left(-\mathrm{i} 2 p x^{\prime}\right) \mathrm{d} x^{\prime},
$$

where $|x\rangle$ is an eigenstate of $\widehat{x}$ and

$$
\left\langle x|\hat{\rho}| x^{\prime}\right\rangle=\sum_{\psi} P_{\psi} \psi(x) \psi^{*}\left(x^{\prime}\right)
$$

where $P_{\psi}$ are the weighting probabilities for various pure states. The Wigner function is a joint quasi-probability distribution and is especially suited for calculating statistical moments of the rotated quadrature operators defined by (2). In the case of a pure state there is a unique correspondence betwcen the wave function $\psi(x)=\langle x \mid \psi\rangle$ and the Wigner function through the relation $\left\langle x|\hat{\rho}| x^{\prime}\right\rangle=\psi(x) \psi^{*}\left(x^{\prime}\right)$.

Furthermore, the probability distribution $P_{\theta}\left(x_{\theta}\right)$ for any quadrature operator $\widehat{x}_{\theta}$ can be obtained by integrating the Wigner function over the conjugate variable $p_{\theta}$,

$$
P_{\theta}\left(x_{\theta}\right)=\int_{-\infty}^{\infty} W\left(x_{\theta} \cos \theta-p_{\theta} \sin \theta, x_{\theta} \sin \theta+p_{\theta} \cos \theta\right) \mathrm{d} p_{\theta} .
$$

To invert (8) we must determine $P_{\theta}\left(x_{\theta}\right)$ for a certain number of phase angles 0 . For simple, smooth-shaped Wigner functions, such as Gaussians, a small number (as few as ten) of phase values are required [9]. For more structured Wigner functions, more angles are needed. We use the filtered back-projection algorithm [6] to carry out the inverse Radon transform to yield $W(x, p)$. Then $(6)$ can be inverse Fourier transformed to obtain the density matrix $\left\langle x+x^{\prime}|\hat{\rho}| x-x^{\prime}\right\rangle$. This fully determines the state of the ensemble of signal pulses.

\section{Colherent-state measurements}

Figure 3 shows the Wigner function measured for a coherent state of the field [4]. This field is obtained by beam splitting a coherent laser pulse and attenuating one of the beams down to the order of a few photons on average. The other beam is used as the local oscillator. It is centered near $x=0, p=3$, corresponding to a mean photon number of 4.5 .

Using the Wigner functions measured in this way, the density matrix can be determined using (6). For these cohcrent-state fields we anticipate the state to be pure. To test this, we calculate $\operatorname{Tr}\left(\widehat{\rho}^{2}\right)$ for the measured $\hat{\rho}$ and find it to be equal to $1.00 \pm 0.02$. This indicates that the states are pure to a high degree of accuracy. Given this we can then determine the complex wave function in the 


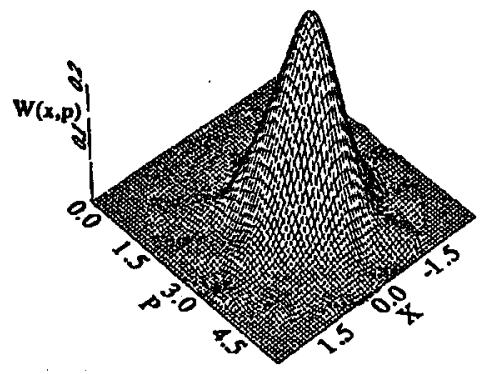

Fig. 3. The Wigner function of a coherent state with a mean photon number of 4.5.
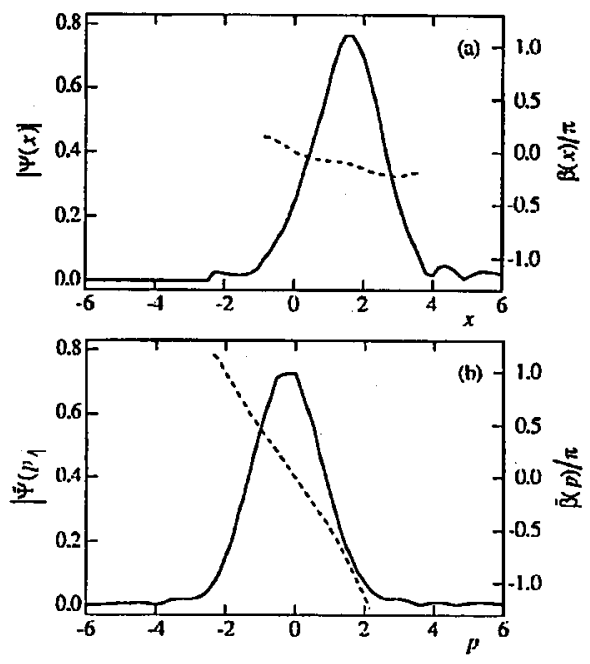

Fig. 4. The measured wave function of a coherent state containing an average of 1.2 photons in (a) the $x$-quadrature representation and (b) the $p$-quadrature representation. The solid line is the magnitude $|\Psi|$ of the wave function and is plotted against the left axis, while the dashed line is the phase $\beta$ of the wave function, plotted against the right axis.

$x$ representation, or in the $p$ representation. Examples of these measured wave functions are shown in Fig. 4 for a coherent state with mean photon number 1.2. The magnilude is seen to have a near-Gaussian form and the phase is constant or linearly changing depending on representation, as expected from elementary theory of coherent states.

\section{Determination of optical phase distributions}

From the type of measurements discussed above, the quantum distributions of optical phase and photon number have been obtained. Results have been obtained for vacuum, coherent, squeezed, and other states [3, 4]. Given 
the measured density matrix, we can experimentally obtain (infer) any of the various quantum distributions of optical phase, in particular the Pegg-Barnett phase distribution [10], the Shapiro-Shepard positive-operator mcasure (POM) phase [11], the marginal Wigner distribution [12], and the Vogel-Schleich opcrational phase distribution [13]. We have used this approach to make measurements of the number-phase uncertainty relation for coherent-state fields. The colierent states do not attain the minimum value for the number-phase uncertainty product, as set by the expectation value of the commutator of the number and phase operators. This commutator expectation value is also determined experimentally from the measured state.

\subsection{Pegg-Barnett or Shapiro-Shepard POM phase}

Using the Pegg-Barnett IIermitian phase-operator formalism, or equivalently the Shapiro-Shepard POM phase formalism, one can define a probability distribution for the phase. For a signal mode in a state described by any (physically realizable) density operator $\hat{\rho}$, the probability density for measuring a particular value of the phase can be written in terms of the number-state basis as

$$
P_{\mathrm{PB}, \mathrm{SS}}(\phi)=\frac{1}{2 \pi} \sum_{n, m=0}^{\infty} \mathrm{e}^{\mathrm{i}(m-n) \phi}\langle n|\hat{\rho}| m\rangle .
$$

The number-state matrix elements of $\hat{\rho}$ are found by integrating the experimentally determined $\left\langle x|\hat{\rho}| x^{\prime}\right\rangle$ with IIermite-Gaussian functions. For all of the coherent states we have measured with mean photon number $\bar{n} \leq 8$, the phase distribution described by (9) converges for $n, m \geq 20$.

\subsection{Wigner phase}

Another way to characterize the phase of a field is via the marginal Wigner phase distribution $P_{\mathrm{W}}(\phi)$ [12]. This is defined as the overlap in phase space of the Wigner distribution and a narrow "wedge-shaped" region, giving the probability density for the pliase to be $\phi$ :

$$
P_{\mathrm{W}}(\phi)=\int_{0}^{\infty} W(r \cos \phi, r \sin \phi) r \mathrm{~d} r .
$$

\subsection{Vogel-Schleich operational phase}

A third way to characterize phase can be accomplished directly from the quadrature distributions, without the need to reconstruct the Wigner function [13]. This can be motivated by considering a similar integration over the Wigner function as in (10), but over a narrow "highway-shaped" region extending through the origin and making an angle $\phi$ with the $x$ axis

$$
P_{\mathrm{VS}}(\phi)=\int_{-\infty}^{\infty} W(r \cos \phi, r \sin \phi) \mathrm{d} r=P_{\theta=\phi+\pi / 2}\left(x_{\theta}=0\right),
$$

the latter equality of which follows from $(\delta)$ and shows that the quadrature distributions $P_{\theta}\left(x_{\theta}\right)$ evaluated at $x_{\theta}=0$ are all that is needed to define this phase 
distribution; tomographic reconstruction is not needed. A conceptual limitation of Eq. (11) is that it is periodic in $\pi$ rather than in $2 \pi$ as are (9) and (10). It therefore can lead to an ambiguity in the distribution if some prior knowledge of the form of the distribution is not available.

\subsection{Experimental results}

Using (9)-(11) it is straightforward to calculate the phase distributions for our experimentally measured states. In Fig. 5a we compare the measured Wigner phase distribution with the measured Pegg-Barnett/Shapiro-Shepard phase distribution for coherent states with different amplitudes. It is seen that as the mean

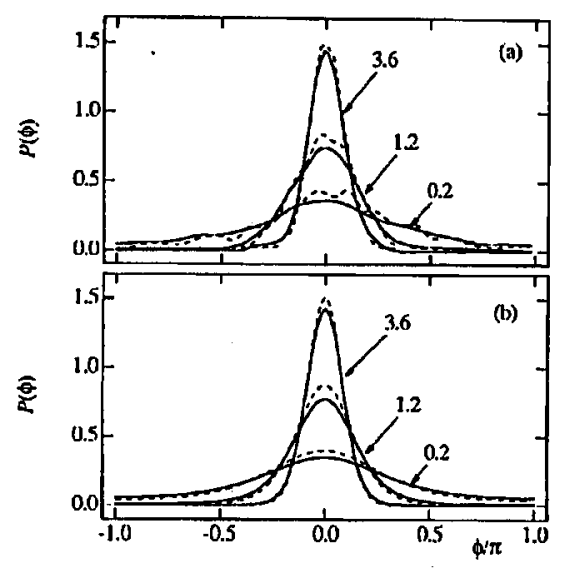

Fig. 5. The solid lines are the Pegg-Barnett/Shapiro-Shepard and the dashed lines are the Wigner phase distributions for experimental (a) and theoretical (b) coherent states. The mean number of photons are indicated.

photon number decreases, the phase distribution broadens, as would be expected from the uncertainty principle. It is also seen that the Wigner phase distribution is more peaked than the Pegg-Barnett/Shapiro-Shepard, as is expected from previous theoretical results [14], and experiments on squeezed states [3]. Shown in Fig. $5 \mathrm{~b}$ are the theoretical phase distributions for coherent states with the same mean photon number as in Fig. 5a. The theoretical results are obtained from Eqs. (9) and (10) with the known theoretical density matrix or the Wigner function for the coherent states inserted.

Figure 6 shows the measured and theoretical operational phase distributions for a coherent state with mean photon number 3.6. For comparison the Pegg-Barnett/Shapiro-Shepard and Wigner phase distributions are also plotted as solid and dotted lines respectively. Note the $\pi$ periodicity in the operational phase distribution. The experimental results are represented as filled squares. 


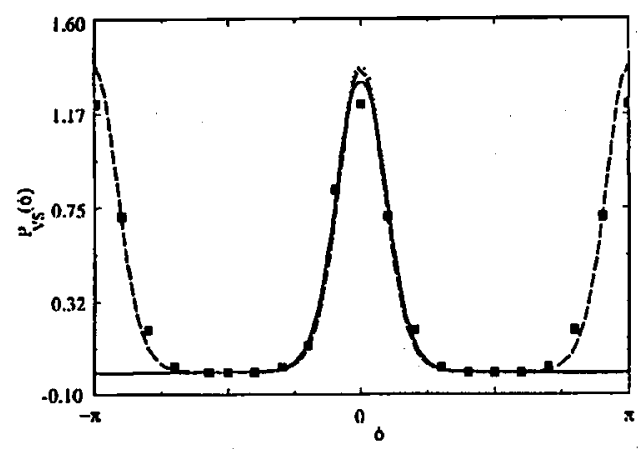

Fig. 6. The measured (points) and theoretical (dashed line) operational phase distributions for a coherent state with a mean number of photons of 3.6. The PeggBarnett/Shapiro-Shepard (solid line) and Wigner (dotted line) phase distributions are also plotted.

\section{Number-phase uncertainty relations}

Because photon number and field phase are conjugate variables, their standard deviations obey an uncertainty relation. The lower bound for the product of standard deviations depends on the state of the field, as has been shown by two different approaches. Pegg and Barnett used a truncated-Hilbert space definition of a Hermitian phase operator $\widehat{\phi}$ to write the uncertainty relation for the phase $\widehat{\phi}$ and photon number $\hat{n}$ operators in the standard operator form

$$
\Delta \phi \Delta n \geq \frac{1}{2}|\langle\Psi|[\hat{\phi}, \widehat{n}]| \Psi\rangle|
$$

where the phase variance is $\Delta \phi^{2}=\left\langle\Delta \widehat{\phi}^{2}\right\rangle=\left\langle\widehat{\phi}^{2}\right\rangle-\langle\hat{\phi}\rangle^{2}$, and $\Delta n^{2}$ is defined similarly, where the expectation values are taken over the state of the system $|\Psi\rangle$. The commutator $[\widehat{\phi}, \hat{n}]$ is an operator, and its expectation value is in general state dependent. Thus, to set the lower bound of experimental measurements of $\Delta \phi \Delta n$, one needs a way to determine the expectation value of the right hand side of (12) for the state of interest. In terms of number states, the commutator can be written [10]:

$$
[\widehat{\phi}, \hat{n}]=\frac{2 \pi}{s+1} \sum_{n=0}^{s} \sum_{n^{\prime} \neq n}^{s}\left|n^{\prime}\right\rangle\langle n| \frac{\left(n-n^{\prime}\right) \exp \left[\mathrm{i}\left(n^{\prime}-n\right) \phi_{0}\right]}{\exp \left[\mathrm{i}\left(n-n^{\prime}\right) 2 \pi /(s+1)\right]-1},
$$

where $s$ is an integer that tends to infinity.

The experimental standard deviation of the phase $\Delta \phi$ is calculated from the Pegg-Barnett distribution (9). The experimental number standard deviation $\Delta n$ is calculated by converting the necessary operator expectation values to Weyl-ordered form and using the Wigner function to carry out the integrals $[4,8]$.

In Fig. 7 are plotted both the uncertainty product $\Delta \phi \Delta n$ and the expectation value of the commutator $\frac{1}{2}|\langle[\hat{\phi}, \widehat{n}]\rangle|$ for our experimentally measured data. 


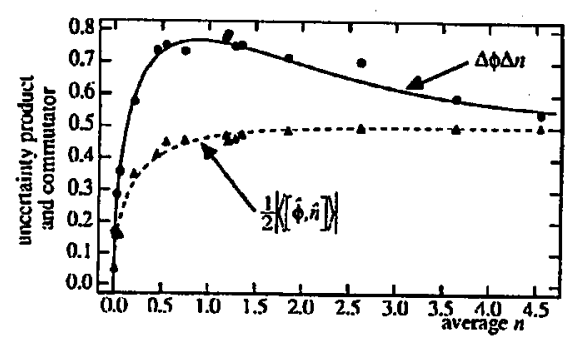

Fig. 7. The number-phase uncertainty product and the expectation value of the number-phase commutator are plotted versus the mean number of photons in a coherent state. The circles are experimentally determined values for the uncertainty product. The triangles are experimentally determined values for the commutator, using either the right-hand side of (12) or (14), which give the same results. The curves are theoretical values for the uncertainty product (solid line) and the commutator (dashed line).

For the experimental data, the commutator expectation value is calculated numerically by expressing the matrices for $\widehat{\phi}$ and $\widehat{n}$ in the number-state-representation, evaluating the matrix which corresponds to the commutator, and tracing the commutator over the density matrix in the number-state-representation. Also plotted are the theoretically predicted values for coherent states. For the theoretical uncertainty product, $\Delta \phi$ was calculated in the same fashion as used to generate Fig. 6 , while the photon number standard deviation was calculated using $\Delta n=\sqrt{\bar{n}}$ for a coherent state.

Notice that the uncertainty relation is satisfied (as it must be using our method of analysis); the uncertainty product is greater than the expectation value of the commutator. It is interesting, however, that the equality between these two quantities is only achieved for average photon numbers approaching zero or approaching a very large number. This is true for both the theoretical and experimental data, so it is not simply a manifestation of our measurements.

Second, we consider the Shapiro-Shepard POM phase, in which no IIermitian phase operator is needed. There the uncertainty relation is derived by using wave functions for the state in number or phase representations, and using the discrete-Fourier transform relation between these representations. The uncertainty relation is found to be [11]:

$$
\Delta \phi \Delta n \geq \frac{1}{2}\left[1-2 \pi P_{\mathrm{PB}, \mathrm{SS}}\left(\phi_{\text {edge }}\right)\right],
$$

where $P_{\mathrm{PB}, \mathrm{Ss}}\left(\phi_{\text {edge }}\right)$ is the probability evaluated at the edge of the chosen phase window. We choose the window so that the peak probability lies at its center. Therefore

$P_{\mathrm{PB}, \mathrm{SS}}\left(\phi_{\text {edge }}\right)$ becomes smaller as the phase becomes better localized around its average - in this limit the right-hand side of (14) approaches the simple Dirac estimate of $1 / 2$. It is known that the right-hand sides of (14) and (12) are exactly equal $[10,11]$. 
We have evaluated the right-hand side of (14) directly from the measured distributions in Fig. 5. The resulting points lie within $1 \%$ of the commutator points shown in Fig. 7 ; this is within the precision with which we can determine these quantities. This result shows that one may use either calculational teclinique (Eq. (12) or (14)) to evaluate the uncertainty lower bound from experimental data.

\section{References}

[1] D.T. Smithey, M. Beck, A. Faridani, M.G. Raymer, Phys. Rev. Lett. 70, 1244 (1993).

[2] D.T. Smithey, M. Beck, J. Cooper, M.G. Raymer, A. Faridani, to appear in Phys. Scr., 1993.

[3] M. Beck, D.T. Smithey, M.G. Raymer, Phys. Rev. A 48, R890 (1993).

[4] M. Beck, D.T. Smithey, J. Cooper, M.G. Raymer, Opt. Lett. 18, 1259 (1993); and Phys. Rev. A, accepted for publication, 1993.

[5] K. Vogel, H. Risken, Phys. Rev. A 40, 2847 (1989).

[6] G.T. Herman, Image Reconstruction from Projections: the Fundamentals of Computerized Tomography, Academic Press, New York 1980.

[7] D.T. Smithey, M. Beck, M. Belsley, M.G. Raymer, Phys. Rev. Lelt. 69, 2650 (1992).

[8] M. Hillery, R.F. O'Connell, M.O. Scully, E.P. Wigner, Phys. Rep. 106, 121 (1984).

[9] A. Faridani, in: Inverse Problems and Imaging; Pitman Research Notes in Mathematics, Vol. 245, Ed. G.F. Roach, Wiley, Essex 1991, p. 68; and private communication.

[10] D.T. Pegg, S.M. Barnett, Phys. Rev. A 39, 1665 (1989).

[11] J.H. Shapiro, S.R. Shepard, Phys. Rev. A 43, 3795 (1991); J.H. Shapiro, in: Proc. Workshop on Squeezed States and Uncertainty Relations, Eds. D. Han et al., NASA Conference Publication 3135, 1992, p. 107.

[12] W. Schleich, R.J. Horowicz, S. Varro, Phys. Rev. A 40, 7405 (1989).

[13] W. Vogel, W. Schleich, Phys. Rev. A 44, 7642 (1991).

[14] G.S. Agarwal, S. Chaturvedi, K. Tara, V. Srinivasan, Phys. Rev. A 45, 4904 (1992). 